\title{
ANÁLISE COMPARATIVA DA QUALIDADE EM DOIS MANEJOS DE SECAGEM EM UM PROCESSO DE BENEFICIAMENTO DE ARROZ
}

\author{
Cleiton Candido Patrício ${ }^{1}$
}

RESUMO: O arroz é alimento presente diariamente na mesa de todos os cidadãos brasileiro, podemos dizer que é a cultura com maior potencial de adaptabilidade a diferentes condições de clima e solo, em função disso, é uma das culturas com maior potencial de combate a fome no mundo. Seu ciclo de produção entre plantio e colheita fica entre 120 e 150 dias dependendo da variedade, após maturo, este produto é colhido por máquinas agrícolas e expedido para armazém, no recebimento no setor de armazenagem, inicia o processo de pre-limpeza para retira de impurezas grosseiras e após seguir para silo armazenador e iniciar processo de secagem. O presente trabalho tem por intuito comprovar o melhor manejo de enchimento para secagem a baixa temperatura entre os anos 2019 e 2020 priorizando a qualidade do grão, buscando o menor percentual possível de grãos amarelos. Para obtenção destas analises, se fez uso do software de apoio minitab, onde através do mesmo, por meio de cartas de controles é possível avaliar se o processo esta dentro ou fora da normalidade. Desta forma pretendesse identificar que o manejo de enchimento adotado implica diretamente na qualidade do produto final, ocasionando perdas no processo industrial de beneficiamento de arroz branco.

Palavras-chave: Arroz. Secagem a baixa temperatura. Software minitab. Qualidade.

ABSTRACT: Rice is food present daily at the table of all Brazilian citizens, we can to say that it is the culture with the greatest potential for adaptability to different climate conditions and soil, depending on this, is one of the crops with the greatest potential to fight hunger in the world. Its production cycle between planting and harvesting is between 120 and 150 days depending on the variety, after maturing, this product is harvested by agricultural machinery and dispatched to warehouse, at the receipt in the storage sector, starts the pre-cleaning process for remove from coarse impurities and after proceed to storage silo and start process drying. The present work aims to prove the best filling handling for low-temperature drying between 2019 and 2020 prioritising grain quality, seeking the lowest possible percentage of yellow grains. To obtain these analyses, it was made use of the support software Minitab, where through the same, by means of control letters is possible to evaluate if the process is within or outside normal. Thus, it intended identify that the filling management adopted directly implies the quality of the product resulting in losses in the industrial processing of white rice.

Keywords: Rice. Low temperature drying. Minitab software. Quality.

IMBA em Gestão de Negócios e Estratégia Competitiva. E-mail: cleitoncandidoı@hotmail.com. 


\section{INTRODUÇÃO}

No modelo de secagem a baixa temperatura em silos armazenadores, o manejo de enchimento do silo é de fundamental importância para determinar perdas qualitativas e quantitativas, pois o grão de arroz quando colhido, possui temperatura e umidade altas, onde é preciso altos fluxos de ar para diminuição desses fatores no menor tempo possível, garantindo principalmente menores perdas em termos de qualidade em grãos amarelos, mofados/ardidos e enegrecidos que irão refletir diretamente no produto final após beneficiamento do grão.

Desta forma, propõe-se realizar uma análise comparativa da qualidade comparando perdas de qualidade em grãos armazenados mediante dois manejos de secagem a baixa temperatura por meio de parâmetros de controle de armazenagem do grão, desenvolver roteiro de coleta de dados e analisar os dados coletados por meio de cartas de controle em software específico.

Por meio de dados fornecidos pela empresa em estudo, pretende-se identificar melhor manejo de enchimento de silos para secagem dos grãos no momento do recebimento da safra do ano de 2022. Desta forma será avaliado dados fornecidos por meio de software de cartas de controle para estabelecer parâmetros de enchimento, proporcionando padronização na operação e assim assegurando que todo grão armazenado no período de 12 meses estará dentro das condições adequadas, gerando os menores percentuais possíveis de defeitos no momento do processamento e assegurando a satisfação do cliente final.

O trabalho é oportuno para empresa devido a definição e padronização do modelo de enchimento da unidade em estudo, podendo ser expandido para as demais filiais da empresa e assim viabilizando todo o trabalho, pois os dados a serem estudados estão disponíveis no banco de dados da unidade armazenadora.

\section{REVISÃO BIBLIOGRAFICA}

\section{I Controle estatístico de processo}

O controle estatístico de processo ou CEP é uma ferramenta da qualidade utilizada como prevenção e detecção de defeitos nos processos produtivos. Segundo Costa, 
Eprecht e Carpinetti, (2004), visa conhecer o processo, estabilizar e fazer os ajustes necessários, pois o monitoramento só ocorre depois que o processo estiver sob controle.

O processo está sob controle, quando está somente sujeito à ação das causas aleatórias (comuns). Quando além das causas aleatórias estiverem presentes causas especiais, ele está fora de controle. Quando o processo está fora de controle e alguma ação corretiva é necessária, um ponto está acima do LSC ou abaixo do LIC no gráfico (COSTA; EPPRECHT; CARPINETT, 2004).

As causas comuns não podem ser evitadas, e quando o processo apresenta somente causas de variação comuns, as variáveis do processo seguem uma distribuição normal. Já as causas especiais podem ser eliminadas e são ocasionadas por motivos claramente identificáveis e alteram os parâmetros do processo, média e desvio padrão (MARTINS; LAUGENI, 2005).

O controle permanente dos processos é condição básica para a manutenção da qualidade de bens e de serviços. Não existe na literatura uma definição única e universal para qualidade (COSTA, EPPRECHT e CARPINETTI, 2005).

Para Toledo, Borrás, Merulhão e Mendes, (2013), a inspeção da qualidade faz-se em um produto já existente, ou seja, já produzido, que pode ser uma matéria prima, um produto em processo ou um produto acabado com a finalidade de verificar se a qualidade do lote atende aos padrões ou especificação de aceitação.

De acordo com Montgomery (2004), é recomendado tomar 20 a 25 amostras, para construção dos limites de controle tentativos, com o objetivo de testar se o processo está estável. Estes dados podem ser obtidos através de dados históricos do processo.

Conforme Mello, Silva, Turrioni e Sousa (2007), uns dos requisitos da norma ISO 9001:200o dispõe, de maneira geral, dos motivos pelos quais a organização deve planejar e implementar os processos de monitoramento, medição, análise e melhoria. São eles:

- Demonstrar a conformidade do produto ou serviço;

- Assegurar a conformidade do sistema de gestão da qualidade;

- Melhorar continuamente a eficácia do sistema de gestão da qualidade; 
Para atender estes objetivos, a organização deve avaliar a necessidade de adequação de técnicas estatísticas em consonância com o seu processo, possibilitando um meio muito eficaz para o desenvolvimento de novas tecnologias e para o controle da qualidade em processos de realização de produtos e serviços, a fim de reduzir sua variabilidade.

Montgomery (2009) define a qualidade como um conjunto de características que tornam um bem ou serviço plenamente adequado ao uso para o qual foi concebido.

\subsection{Cartas de controle}

A literatura cita inúmeros estudos relacionando a utilização de métodos de Controle Estatístico de Processo (CEP), tais como em gerenciamento de processos ou de sistemas produtivos, destinados a monitorar a estabilidade e acompanhar seus parâmetros ao longo do tempo (ROSA, 2009).

Com a utilização de ferramentas do CEP, como por exemplo, o uso de gráficos de controle, é possível fornecer informações como a capacidade e a estabilidade do processo produtivo (PABLE, LU, \& AUERBACH, 2ого).

Rai (2008), realizou um estudo aplicando controle estatístico do processo, numa indústria de fabricação de chás, utilizando as cartas de controle do tipo CUSUM e da X Bar. O objetivo era resolver o problema de variação da característica de qualidade "peso", na hora do empacotamento. Como resultados alcançados, conseguiram reduzir a variação com taxa de $66 \%$ para $4 \%$.

Lim (2014) em sua pesquisa, mostra que a indústria de alimentos carece de trabalhos que apliquem o Controle Estatístico do Processo em seu processo produtivo. Como sugestões de pesquisas futuras Lim (2014) comenta que existe uma lacuna para trabalhos que desenvolvam um método prático que facilite e operacionalize a implantação do Controle Estatístico do Processo na indústria de alimentos.

Segundo Montgomery (2009) é realizada uma análise retrospectiva dos dados que servem basicamente para definir o estado atual da produção, e definir os limites de controle que poderão ser usados para o monitoramento futuro do processo.

De acordo com o manual da (AIAG, 2005), para se construir uma carta de controle, deve-se coletar amostra de dados que representem bem o processo estudado, 
assim sendo, quanto maior for a amostra, maior será a chance de detectar pequenas mudanças no processo.

\subsection{Cartas de controle X-AM}

De acordo com Montgomery (2004); Minitab (2018), a carta I-AM é usada para monitorar a média e a variação do seu processo quando se tem dados contínuos que são observações individuais e não em subgrupos. Esta carta de controle tem o propósito de monitorar a estabilidade do processo ao longo do tempo para que seja possível identificar e corrigir as instabilidades nele.

Há situações em que o tamanho da amostra usada para avaliação do controle do processo é $\mathrm{n}=\mathrm{I}$. Por exemplo, na fabricação de aço, celulose e outros elementos químicos, o controle do processo é realizado retirando-se amostras de uma unidade para se medir $\mathrm{PH}$, viscosidade, como não é possível estimar a variabilidade através da amplitude ou do desvio padrão de cada amostra, usa-se como estimativa da variabilidade a amplitude móvel de duas (ou mais) observações sucessivas (MONTGOMERY, 2004).

Segundo Montgomery (2004), as equações válidas para a análise dos valores individuais e amplitudes móveis, respectivamente são:

$$
\begin{aligned}
& L C=\bar{X} \\
& L I C=\bar{X}-\left(\frac{3 * \overline{M R}}{d_{2}}\right)=\bar{X}-\left(E_{2} * \overline{M R}\right) \\
& L S C=D_{4} * \overline{M R} \\
& L C=\overline{M R} \\
& L I C=D_{3} * \overline{M R}
\end{aligned}
$$

\subsection{Cartas de controle Xbar- $\mathrm{R}$}

De acordo com Pignatiello Jr. e Simpson (2002); Toledo et al. (2013), destacam-se os gráficos de controle X-bar/S-bar (R-bar) são os gráficos mais utilizados na prática.

Previamente Machado e Costa (2008) ressaltam que, o poder deste tipo de gráfico de controle está na sua capacidade de diferenciar as causas assinaláveis das causas 
especiais. Já Santiago e Smith (2013), busca encontrar deslocamentos de parâmetros, alterações na magnitude da variação ou padrões não aleatórios.

Já Costa et al. (2014) afirmam que, quando a variável controlada é contínua, tornase necessário o monitoramento do processo com dois gráficos de controle: um para a centralidade e o outro para a dispersão.

O cálculo dos parâmetros estatísticos do gráfico de controle X-bar/R-bar serão demonstrados pelas equações abaixo (MONTGOMERY, 2009; TOLEDO, 2013).

$$
\text { Média: } \quad \overline{\bar{x}}=\frac{\bar{x}_{1}+\bar{x}_{2}+\ldots+\bar{x}_{m}}{m}
$$

$$
\text { Amplitude média: } \quad \bar{R}=\frac{R_{1}+R_{2}+\ldots+R_{m}}{m}
$$

$$
\begin{array}{llll}
L I C_{\bar{x}}: & \overline{\bar{x}}-A_{2} \bar{R} & \\
& & L S C_{\bar{x}}: & \overline{\bar{x}}+A_{2} \bar{R} \\
\mathrm{~A}_{2}: & \frac{3}{d_{2} \sqrt{n}} & L I C_{R}: & D_{4} \bar{R} \\
& & D_{R}: & D_{3} \bar{R} !
\end{array}
$$

\subsection{Software minitab}

De acordo com manual do Minitab (2003), o software é uma ferramenta do Six Sigma, empregado em todas as fases dos projetos. Trata-se de um software de gerenciamento de processos e estatística com muitos recursos, capaz de calcular e gerar gráficos estatísticos e testes de hipótese para validar amostras e processos com base em análise de amostragem.

Segundo manual Minitab I8 (2019), o software cria gráficos e realiza análises estatísticas que ajudam a chegar no centro de expedição tendo assim o sistema informatizado muito eficiente, com os dados agrupados centro de expedição pode-se criar cartas de controle e testar se o processo está dentro ou fora dos limites de controle 
estabelecidos, por fim, se houver pontos fora, é possível analisar e melhorar o processo para que o mesmo atenda as especificações desejadas.

Para Cymrot, Mani e Lara (2006) o programa estatístico MINITAB teve início em 1972 fundado por uma equipe de professores da Universidade da Pensilvânia para auxiliar no ensino da estatística. Hoje o programa é usado por mais de 4000 faculdades e universidades em todo o mundo. O programa também é utilizado no mundo dos negócios em 80 países do mundo, inclusive em várias empresas brasileiras.

Conforme Teixeira (2019) o software Minitab possui um amplo pacote de ferramentas sobre machine learning, melhorias de processo e análise estatística, podendo ser utilizado em empresas de vários portes, ajudando resolver problemas, identificar tendências, e analisar dados.

Inicialmente, o software é alimentado com informações adquiridos na etapa de coleta de dados realizadas no ambiente de estudo. Após é realizado o estudo de medição $R \& R$ (cruzado) em que é analisado a repetibilidade e reprodutibilidade dos dados, no qual é avaliado a variação do sistema de medição adotado durante a coleta de dados (TEIXEIRA, 2019).

Piratelli-Filho, Zúñiga e Arencibia (2014) afirmam que os parâmetros do estudo de medição $R \& R$ são amplamente utilizados em diversos setores da indústria, de diferentes portes e áreas, tendo como objetivo principal a verificação da adequabilidade do processo de medição, pois é de suma importância avaliar tanto a capacidade do medidor quanto a capacidade do sistema de inspeção para se assegurar a qualidade das medições a serem coletadas no processo.

Em seguida é feito o teste de normalidade dos dados coletados para confirmar a probabilidade de os valores serem coletados em outras medições. Já Ribeiro Júnior (2012), comenta que o teste de normalidade é indicado para a análise de medições contínuas, como de comprimento ou peso, e determina se os dados irão seguir uma distribuição normal. Para realização do teste primeiramente é estabelecido a hipótese de análise e valor $p$, a qual consiste na probabilidade estatística dos resultados incidirem novamente, sendo avaliado por valor de $\alpha$ estabelecido e dependendo deste valor, afirma ou nega hipótese previamente estipulada. 
Logo Shin et al. (2012), afirma que o método mede se os dados coletados seguem uma distribuição específica de maneira ótima. $\mathrm{O}$ teste estatístico $\mathrm{AD}$ é aplicado com finalidade de determinar o valor de p para o ensaio de qualidade do arranjo, que auxilia a designar o que melhor se adaptar-se com a disposição dos dados coletados.

\subsection{Legislação para armazenagem de arroz}

O arroz em casca ou beneficiado, tanto natural como parboilizado, seja ele polido ou integral, é classificado de acordo com normas estabelecidas pelo MAPA (Ministério da Agricultura, Pecuária e Abastecimento). Atualmente, as normas em vigor (IN oo6/2009 de I8/02/2009 e IN 002/2012 de 07/02/2012) estão adequadas às normas internacionais, principalmente aos tratados do Mercosul, o que propicia um produto com melhor qualidade para o consumidor (EIFERT, SILVA e COSTA, 2021) (EMBRAPA CULTIVO DO ARROZ, 202I).

As normas definem critérios de qualidade para cada tipo de arroz, estabelecendo a classe, dado pelas dimensões dos grãos, longo, longo fino, médio e curto, e o tipo do arroz, por meio da quantificação: defeitos graves, percentuais de grãos pretos, ardidos e mofados, e os defeitos gerais agregados - DGA, percentuais de grãos manchados e picados, rajados, verdes e gessados e amarelos. Essas definições de defeitos são para arroz em casca, natural ou parboilizado. Para o arroz beneficiado, agregam-se a esses critérios o percentual de quebrados e de impurezas, entre outros. Portanto, é cada vez mais importante concentrar esforços durante a condução da lavoura para minimizar defeitos nos grãos, diminuindo assim perdas qualitativas e, consequentemente, reduzindo o risco de grandes perdas econômicas (EIFERT, SILVA e COSTA, 202I) (EMBRAPA - CULTIVO DO ARROZ, 2021).

\subsection{Classificação do arroz polido}

As análises de classificação de arroz realizadas no processo da indústria/armazém de arroz polido, são de controle interno, porém para que se atinja o objetivo final que é o enquadramento do produto dentro das legislações vigentes estabelecidas pelo Ministério da Agricultura, Pecuária e Abastecimento- MAPA, se faz necessário ter um controle rigoroso do processo de recebimento, armazenagem e processamento do produto. 
De acordo com a Instrução Normativa no 6, de i6 de fevereiro de 2009 do MAPA, a classificação do arroz será estabelecida em função dos seus requisitos de identidade e de qualidade, sua comercialização se dá pela tipificação, para que o produto se enquadre como tipo I, classe longo fino polido, obedecendo os critérios estabelecidos apresentados na figura 02.

Figura 02 - Arroz Beneficiado Polido

Arroz Beneficiado Polido - Limites máximos de tolerância expressos em \%/peso

\begin{tabular}{|c|c|c|c|c|c|c|c|c|}
\hline Tipo & $\begin{array}{l}\text { Maté } \\
\text { Estra } \\
\text { e Imp } \\
\text { Impu }\end{array}$ & $\begin{array}{l}\text { Mofados } \\
\text { Ardidos }\end{array}$ & $\begin{array}{r}\text { ePicad } \\
\text { Manc }\end{array}$ & $\begin{array}{l}\text { Gessado } \\
\text { e Verdes }\end{array}$ & ${ }^{S}$ Rajados & Amarelos & \multicolumn{2}{|c|}{$\begin{array}{l}\text { Total de } \\
\text { Quebrados Quirera(máximo) } \\
\text { e Quirera }\end{array}$} \\
\hline 1 & 0,10 & 0,15 & 1,75 & 2,00 & 1,00 & 0,50 & 7,50 & 0.50 \\
\hline 2 & 0,20 & 0,30 & 3,00 & 4,00 & 1,50 & 1,00 & 15,00 & 1,00 \\
\hline 3 & 0,30 & 0,50 & 4,50 & 6,00 & 2,00 & 2,00 & 25,00 & 2,00 \\
\hline 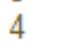 & 0,40 & 1,00 & 6,00 & 8,00 & 3,00 & 3,00 & 35,00 & 3,00 \\
\hline 5 & 0,50 & 1,50 & 8,00 & 10,00 & 4,00 & 5,00 & 45,00 & 4,00 \\
\hline
\end{tabular}

Observação: O limite máximo de tolerância admitido para marinheiro é de 10 (dez) grãos em $1000 \mathrm{~g}$ (um mil gramas) para todos os tipos. Acíma desse limite o produto será considerado como Fora de Tipo.

Fonte: Brasil (2009).

Se ultrapassar algum destes limites o produto se enquadra como fora de tipo, podendo assim ser comercializado apenas para alimentação animal.

Descrição dos indicadores de classificação: (Brasil 2009).

Arroz tipo I classe longo fino: o produto que contém, no mínimo, 80\% (oitenta por cento) do peso dos grãos inteiros medindo 6,00 $\mathrm{mm}$ (seis milímetros) ou mais no comprimento, a espessura menor ou igual a $1,90 \mathrm{~mm}$ (um vírgula noventa milímetros) e a relação comprimento/ largura maior ou igual a 2,75 (dois vírgulas setenta e cinco), após o polimento dos grãos;

Arroz fora de tipo: o produto que exceder os limites máximos de tolerância estabelecidos para o Tipo 5.

Matérias estranhas e impurezas: os corpos ou detritos de qualquer natureza estranhos ao produto, a exemplo dos grãos ou sementes de outras espécies vegetais, sujidades e insetos, os detritos do próprio produto, a exemplo da casca do arroz (aberta), dos grãos chochos e dos pedaços de caule;

Umidade: $O$ percentual de umidade tecnicamente recomendado para comercialização do arroz beneficiado, das variedades especiais de arroz, dos fragmentos de 
arroz e do arroz com premix, de qualquer dos subgrupos de arroz beneficiado, bem como da mistura de arroz polido e parboilizado, será de I4\% (catorze por cento).

Grão mofado: o grão descascado ou descascado e polido, inteiro ou quebrado, que apresentar contaminações fúngicas (mofo ou bolor) visíveis a olho nu;

Grão ardido: o grão descascado e polido, inteiro ou quebrado, que apresentar, no todo ou em parte, coloração escura proveniente do processo de fermentação; igualmente são considerados grãos ardidos:

a) o grão descascado e polido, inteiro ou quebrado, que apresentar alteração na sua coloração normal, de marrom escuro a parcialmente preto, resultante do processo de fermentação;

b) o grão ou o fragmento de grão, totalmente preto, encontrado no arroz beneficiado integral e no arroz beneficiado polido;

c) no arroz beneficiado polido, o grão que apresentar coloração escura em mais de $1 / 4$ (um quarto) da sua área;

d) no arroz parboilizado, o grão amarelo destoante de tom escuro (amarronzado ou avermelhado);

Grão enegrecido: o grão descascado e polido, inteiro ou quebrado que se apresentar enegrecido em toda a sua superfície por ação excessiva do calor no processo de parboilização;

Grão não gelatinizado: o grão inteiro ou quebrado que não apresenta gelatinização do amido, devido à parboilização deficiente, mostrando-se totalmente opaco sob a luz polarizada;

Grão danificado: o grão descascado e polido, inteiro ou quebrado, que, pelo processo de parboilização, estoura (pipoca) ou apresenta rachaduras no sentido longitudinal, excetuado o grão com pequenas rachaduras, desde que seu formato não seja alterado;

Grão rajado: o grão descascado e polido, inteiro ou quebrado, que apresentar qualquer ponto ou estria vermelha ou preta, destoante da variedade predominante; no arroz das variedades especiais, de pericarpo vermelho ou preto, o grão rajado não será considerado defeito; 
Grão manchado ou picado: o grão descascado e polido, inteiro ou quebrado, que apresentar mancha escura ou esbranquiçada, perfurações ou avarias provocadas por pragas ou outros agentes, desde que visíveis a olho nu, bem como as manchas escuras provenientes de processo de fermentação em menos de 1/4 (um quarto) da área do grão:

a) as minúsculas perfurações denominadas alfinetadas ou Peck não serão consideradas defeitos; e

b) no arroz parboilizado polido e parboilizado integral serão considerados manchado o grão que apresentar coloração amarelo-escura a marrom;

Grão quebrado: o pedaço de grão de arroz descascado e polido que apresentar comprimento inferior às $3 / 4$ (três quartas) partes do comprimento mínimo da classe que predomina e que ficar retido na peneira de furos circulares de $\mathrm{I}, 60 \mathrm{~mm}$ (um vírgula sessenta milímetros) de diâmetro;

Quirela: o fragmento de arroz que vazar na peneira de furos circulares de $1,60 \mathrm{~mm}$ (um vírgula sessenta milímetros) de diâmetro.

A seguir serão apresentados os procedimentos metodológicos a serem aplicados na pesquisa.

\section{PROCEDIMENTOS METODOLÓGICOS}

\section{I Delineamento da pesquisa}

O delineamento da pesquisa se caracteriza como pesquisa aplicada devido a comparação de dois métodos de manejo de armazenagem com dados já existente, coletados nos anos de 2019 e 2020, onde foram aplicados os dois manejos de secagem em um processo de beneficiamento de arroz. Desta forma, a pesquisa classifica-se quanto aos fins como pesquisa-ação e quanto aos meios como estudo de caso.

Para Koshy, Koshy e Waterman, (2010), definem que a metodologia da pesquisaação envolve participantes conduzindo inquéritos sistemáticos com a finalidade de ajudálos a melhorar as suas próprias práticas, que por sua vez, podem também melhorar o seu ambiente de trabalho e os ambientes de trabalho das pessoas que fazem parte dela. 


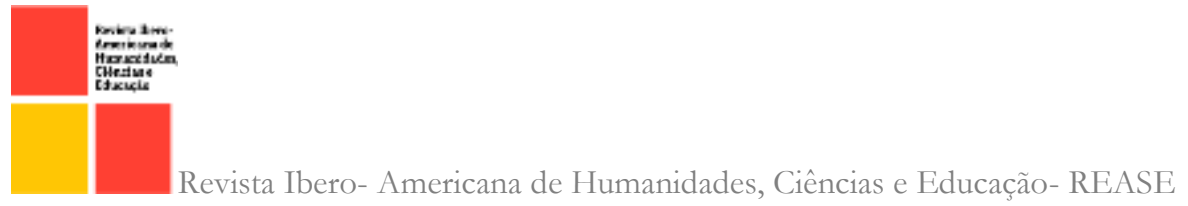

Segundo Thiollent, (20II), a pesquisa-ação tem por finalidade possibilitar aos sujeitos da pesquisa, participantes e pesquisadores, os meios para conseguirem responder aos problemas que vivenciam com maior eficiência e com base em uma ação transformadora. Ela facilita a busca de soluções de problemas por parte dos participantes, aspecto em que a pesquisa convencional tem pouco alcançado.

A metodologia do estudo de caso revela a carência de amadurecimento metodológico dos pesquisadores. Isso contribui para o desenvolvimento lento de pressupostos teóricos mais robustos, para a continuidade de métodos descritivos e pouco envolvidos com as peculiaridades e contextos naturais dos fenômenos que o estudo de caso pretende complementar (BARBOSA, 2008).

Também Flick (2004) afirma que adotar a prática de combinar análise quantitativa e qualitativa proporciona maior nível de credibilidade e validade aos resultados da pesquisa, o que poderia evitar o reducionismo por uma opção única de análise.

\subsection{Local do estudo}

O local de estudo foi a empresa onde o pesquisador atua, no setor de armazenagem de arroz em casca, por necessidade de padronizar o manejo de armazém de uma das unidades.

\subsection{Plano de coleta de dados}

Quanto ao plano de coleta de dados foi composto de dados secundários fornecidos pela empresa em estudo.

\subsection{Plano de análise de dados}

A pesquisa foi realizada sobre uma abordagem quantitativa no banco de dados existente. 


\section{ANÁlisE DOS RESULTADOS}

O estudo tem como foco identificar melhor manejo de enchimento de silos para secagem dos grãos no momento do recebimento da safra do ano de 2022.

Para isso, foram desenvolvidas cartas de controle com base em processos distintos desenvolvidos no ano de 2019 e 2020 .

Os dados foram inseridos no software minitab para estabelecer parâmetros de enchimento, proporcionando padronização na operação e assim assegurando a satisfação do cliente final.

A variável analisada foi a umidade do grão armazenado que afeta diretamente o percentual de grãos amarelos (limite de $0,5 \%$ ), o percentual de grãos mofados, ardidos ou pretos (limite de $0,15 \%$ ) e o indicador de brancura do grão como pode ser visualizado na tabela I:

Tabela I - Indicadores dos Grãos por Silo (2019)

\begin{tabular}{|c|c|r|r|r|r|}
\hline 2019 & AMOSTRAS/SILO & UMIDADE & AMARELO & MOF/ARD/PT & BRANCURA \\
\hline DESC.SILO 01 SAP & 20 & 12,31 & 0,11 & 0,02 & 37,47 \\
\hline DESC.SILO 02 SAP & 9 & 12,13 & 0,09 & - & 43,01 \\
\hline DESC.SILO 03 SAP & 20 & 12,75 & 0,08 & 0,01 & 42,34 \\
\hline DESC.SILO 04 SAP & 17 & 12,51 & 0,23 & 0,02 & 41,73 \\
\hline DESC.SILO 05 SAP & 37 & 11,94 & 0,05 & - & 28,50 \\
\hline DESC.SILO 06 SAP & 31 & 12,57 & 0,22 & 0,01 & 25,06 \\
\hline DESC.SILO 08 SAP & 17 & 11,99 & 0,07 & - & 9,77 \\
\hline DESC.SILO 10 SAP & 22 & 12,06 & - & - & 40,08 \\
\hline DESC.SILO 12 SAP & 24 & 12,73 & 0,02 & 0,01 & 34,03 \\
\hline DESC.SILO 13 SAP & 18 & 12,69 & - & - & 40,51 \\
\hline DESC.SIL0 14 SAP & 18 & 12,82 & 0,09 & 0,01 & 40,94 \\
\hline DESC. SILO 16 SAP & 24 & 13,01 & 0,27 & - & 40,45 \\
\hline DESC. SILO 17 SAP & 24 & 13,01 & 0,11 & - & 40,61 \\
\hline DESC. SILO 18 SAP & 9 & 12,92 & 0,11 & 0,01 & 41,54 \\
\hline DESC. SILO 19 SAP & 19 & 13,07 & 0,37 & - & 40,62 \\
\hline DESC. SILO 20 SAP & 17 & 13,38 & 0,11 & - & 40,93 \\
\hline DESC. SILO 21 SAP & 12 & 14,05 & 0,17 & 0,01 & 42,59 \\
\hline MÉDIA 100\% do Silo & 338 & 12,70 & 0,12 & 0,01 & 37,07 \\
\hline
\end{tabular}

Fonte: O Pesquisador. 
As cartas de controle utilizadas foram as cartas X e AM que monitoram a média e a variação de um processo quando existem dados contínuos e subgrupos.

Primeiramente verificou-se a variação e a média da umidade colocando as amostras no Minitab, dados que podem ser visualizados na figura I:

Figura I - Cartas de controle X - AM (2019)

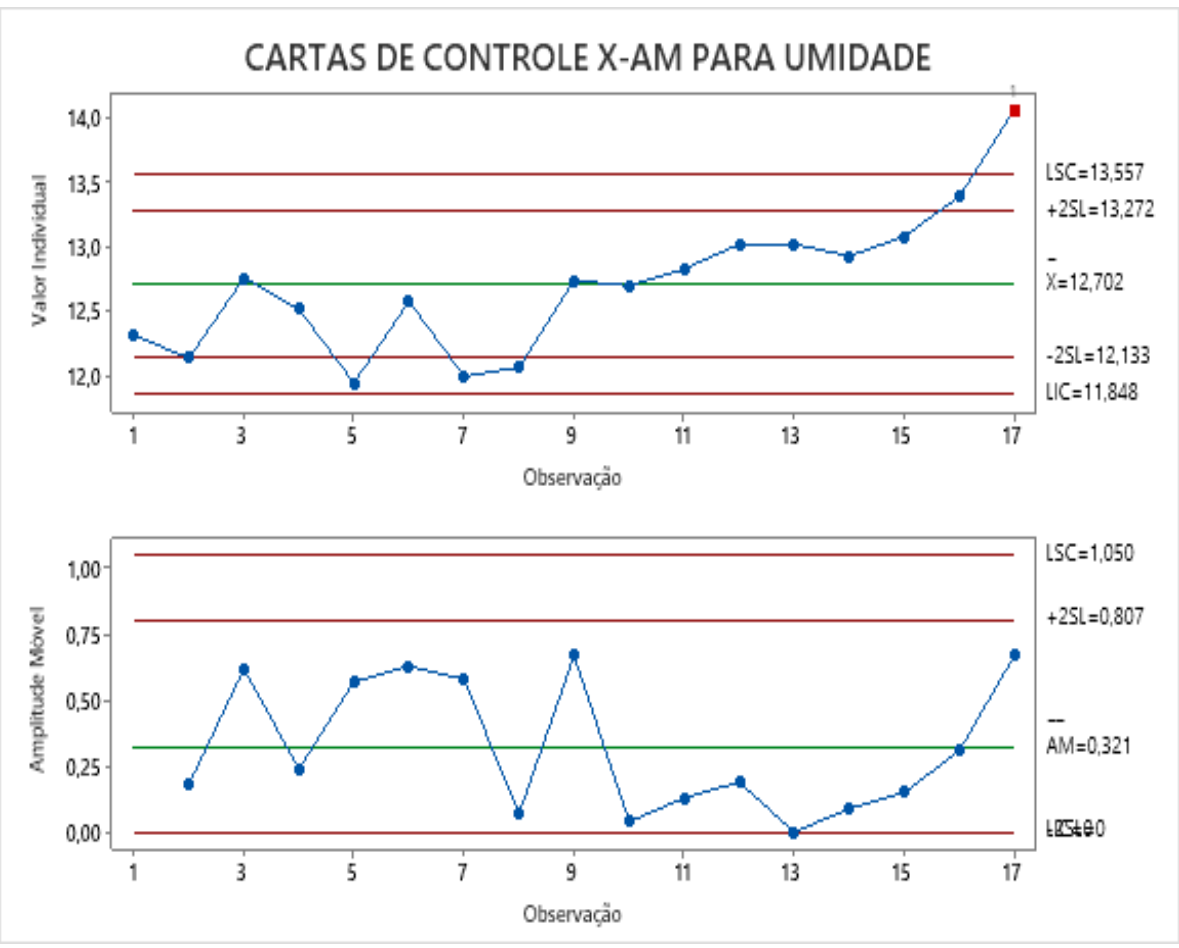

Fonte: $\mathrm{O}$ Pesquisador.

Percebe-se a partir dos dados plotados que a média no ano de 2019 ficou em 12,702, o limite superior de controle em 13,557 e o limite inferior de controle em ir,848. Desta forma, o teste apresenta uma tendência ao limite superior de controle com falha no ponto I7 extrapolando o LSC em mais de três desvios padrão.

Apesar de ter ocorrido a falha no limite superior de controle, observa-se no gráfico de amplitude que captura a variação dentro do subgrupo uma distribuição entre os limites de controle

A seguir, são apresentadas as cartas para as amostras coletadas no ano de 2020 , na tabela 2: 
Tabela 2 - Indicadores dos Grãos por Silo (2020)

\begin{tabular}{|c|c|c|c|c|c|}
\hline 2020 & AMOSTRAS/SILO & UMIDADE & AMARELO & MOF/ARD/PT & BRANCURA \\
\hline DESC.SILO o3 SAP & 16 & 13,04 & 0,02 & - & 43,74 \\
\hline DESC.SILO o4 SAP & 14 & 13,31 & - & - & 45,70 \\
\hline DESC.SILO os SAP & 15 & 12,54 & 0,03 & o,oI & 46,39 \\
\hline DESC.SILO o6 SAP & 46 & $I 2,7 I$ & - & $\mathrm{o}, \mathrm{OI}$ & 45,30 \\
\hline DESC.SILO o7 SAP & 42 & 12,45 & - & $\mathrm{o}, \mathrm{OI}$ & 46, or \\
\hline DESC.SILO o9 SAP & I5 & 13,09 & 0,03 & - & 44,75 \\
\hline DESC.SILO $_{12}$ SAP & 37 & 13,13 & 0,04 & - & $43,4 \mathrm{I}$ \\
\hline DESC.SILO $_{13}$ SAP & 23 & 12,57 & o,Io & $\mathrm{o}, \mathrm{OI}$ & 44,78 \\
\hline DESC.SILO $_{14}$ SAP & 13 & $I 2,44$ & - & o,o6 & 46,02 \\
\hline DESC. SILO I8 SAP & 7 & $I 2,4 I$ & 0,03 & - & $40,5 \mathrm{I}$ \\
\hline DESC. SILO I9 SAP & I4 & 12,15 & 0,02 & $\mathrm{o}, \mathrm{OI}$ & 43,92 \\
\hline DESC. SILO 20 SAP & 2 & 12,55 & 0,03 & - & 40,45 \\
\hline DESC. SILO 21 SAP & 7 & 12,60 & 0,02 & $\mathrm{o}, \mathrm{OI}$ & 38,20 \\
\hline MÉDIA $65 \%$ do silo & $25 \mathrm{I}$ & 12,69 & 0,02 & 0,01 & 43,78 \\
\hline
\end{tabular}

Fonte: O Pesquisador.

A plotagem das amostras em relação a umidade, gerou as seguintes cartas de controle: 
Figura 2 - Cartas de Controle X - AM (2020)

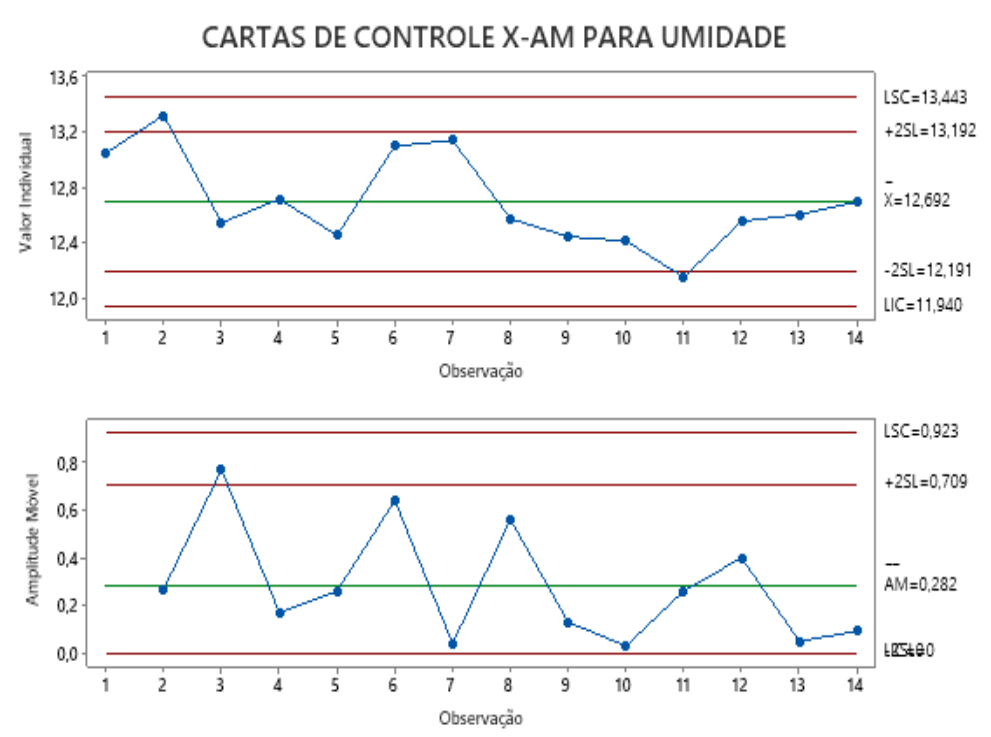

Fonte: O Pesquisador.

A média da umidade no processo ficou em 12,692 sendo os limites superior de controle LSC $=13,443$ e o limite inferior de controle LIC $=$ II,940. Nenhuma das amostras apresentou falha nem nas medias nem na amplitude do processo, demonstrando que ele estava sob controle mesmo com uma tendência ao limite inferior a partir da amostra 8.

O segundo aspecto avaliado no processo foi a verificação comparativa entre os anos de 2019 e 2021 levando em consideração as variáveis: amarelado do grão e mofo/ardido ou preto do grão, das amostras coletadas nos mesmos equipamentos.

Foi realizada uma análise de variância utilizando os dados da tabela 3:

Tabela 3 - Dados Utilizados para Análise de Variância

\begin{tabular}{|l|r|r|r|r|r|r|}
\hline \multirow{2}{*}{ EQUIPAMENTO } & \multicolumn{3}{|c|}{2019} & \multicolumn{3}{|c|}{2020} \\
\cline { 2 - 7 } & UMIDADE & AMARELO & MOF/ARD/PT & UMIDADE & AMARELO & MOF/ARD/PT \\
\hline DESC.SILO 03 SAP & 12,75 & 0,08 & 0,01 & 13,04 & 0,02 & 0,00 \\
\hline DESC.SILO 04 SAP & 12,51 & 0,23 & 0,02 & 13,31 & 0,00 & 0,00 \\
\hline DESC.SILO 05 SAP & 11,94 & 0,05 & 0,00 & 12,54 & 0,03 & 0,01 \\
\hline DESC.SILO 06 SAP & 12,57 & 0,22 & 0,01 & 12,71 & 0,00 & 0,01 \\
\hline DESC.SILO 12 SAP & 12,73 & 0,02 & 0,01 & 13,13 & 0,04 & 0,00 \\
\hline DESC.SILO 13 SAP & 12,69 & 0,00 & 0,00 & 12,57 & 0,10 & 0,01 \\
\hline DESC.SILO 14 SAP & 12,82 & 0,09 & 0,01 & 12,44 & 0,00 & 0,06 \\
\hline DESC. SILO 18 SAP & 12,92 & 0,11 & 0,01 & 12,41 & 0,03 & 0,00 \\
\hline DESC. SILO 19 SAP & 13,07 & 0,37 & 0,00 & 12,15 & 0,02 & 0,01 \\
\hline DESC. SILO 20 SAP & 13,38 & 0,11 & 0,00 & 12,55 & 0,03 & 0,00 \\
\hline DESC. SILO 21 SAP & 14,05 & 0,17 & 0,01 & 12,60 & 0,02 & 0,01 \\
\hline
\end{tabular}

Fonte: O Pesquisador. 
4.I Análise de variância em relação a umidade

Utilizando a ANOVA com fator de umidade versus o método de manejo de enchimento dos silos nos anos 2019 e 2020 percebeu-se que o primeiro não influenciou no indicador de umidade do grão mediantes ao modelo de secagem adotados. Pois o método de secagem a baixa temperatura de arroz é padrão, o que influencia é o tempo que o produto exposto ao ar de secagem.

Tabela 4 - Análise em Relação Umidade

\begin{tabular}{|c|c|c|c|c|c|}
\hline \multicolumn{6}{|c|}{ Método } \\
\hline \multicolumn{2}{|c|}{ Hipótese nula } & \multicolumn{4}{|c|}{ Todas as médias são iguais } \\
\hline \multicolumn{2}{|c|}{ Hipótese alternativa } & \multicolumn{4}{|c|}{ Nem todas as médias são iguais } \\
\hline \multicolumn{2}{|c|}{ Nivel de significância } & \multicolumn{4}{|c|}{$a=0,05$} \\
\hline \multicolumn{6}{|c|}{ Assumiu-se igualdade de variâncias para a análise } \\
\hline \multicolumn{6}{|c|}{ Informações dos Fatores } \\
\hline Fator & Niveis & \multicolumn{4}{|c|}{ Valores } \\
\hline Ano & 2 & \multicolumn{4}{|c|}{$2019 ; 2020$} \\
\hline \multicolumn{6}{|c|}{ Informações de Agrupamento Usando Método de Tukey e $95 \%$ de Confiança } \\
\hline ANO & $\mathrm{N}$ & \multicolumn{2}{|c|}{ Média } & \multicolumn{2}{|c|}{ Agrupamento } \\
\hline 2019 & 11 & \multicolumn{2}{|c|}{12,857} & \multicolumn{2}{|c|}{ A } \\
\hline 2020 & 11 & \multicolumn{2}{|c|}{12,677} & \multicolumn{2}{|c|}{ A } \\
\hline \multicolumn{6}{|c|}{ Médias que não compartilham uma letra são significativamente diferentes. } \\
\hline \multicolumn{6}{|c|}{ Análise de Variância } \\
\hline Fonte & GL & SQ (Aj.) & QM (Aj.) & Valor F & Valor-P \\
\hline Ano & 1 & 0,1782 & 0,1782 & 0,88 & 0,359 \\
\hline Erro & 20 & 4,0428 & 0,2021 & & \\
\hline Total & 21 & 4,221 & & & \\
\hline \multicolumn{6}{|c|}{ GL = Graus de Liberdade } \\
\hline \multicolumn{6}{|c|}{$\mathrm{SQ}$ = Soma dos Quadrados } \\
\hline \multicolumn{6}{|c|}{ QM = Quadrado Médio } \\
\hline \multicolumn{6}{|c|}{$\begin{array}{l}F=\text { Estatística de Teste } \\
P=P \text { valor }\end{array}$} \\
\hline$P=P$ valor & & & & & \\
\hline
\end{tabular}


Como $p>\alpha$ conclui-se que $o$ ano não influenciou na umidade. No gráfico $I$ podemos confirma, que a diferença de manejo adotado para enchimento do silo, não influenciou no indicador de umidade do grão, ou seja, para este indicador, independente do manejo adotado, se atingiu a umidade ideal para armazenagem.

Gráfico I - Intervalos de Umidade x Ano

Gráfico de Intervalos de UMIDADE versus ANO

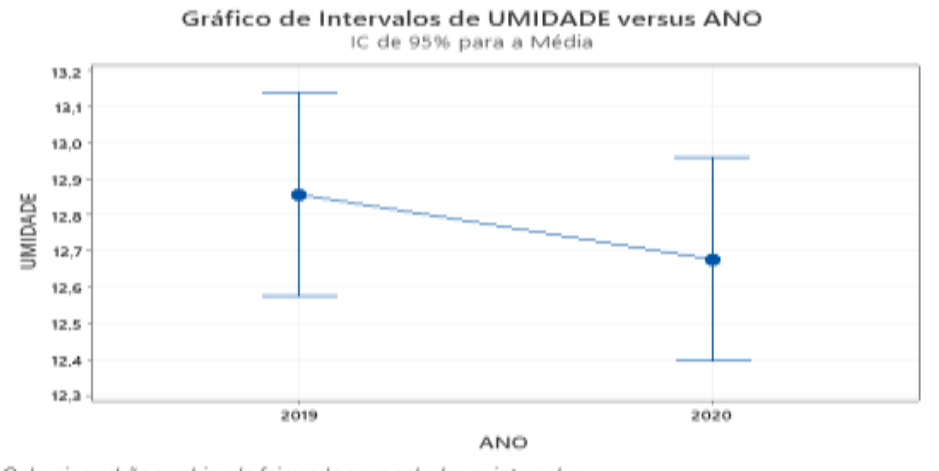

Fonte: O Pesquisador.

\subsection{Em relação ao amarelo}

ANOVA com fator de grão amarelo versus o método de manejo de enchimento dos silos nos anos 2019 e 2020 influenciou na qualidade do grão, pois neste indicador, o que influencia é o tempo de secagem, altura da coluna de grão e fluxo de ar adotado, quanto mais ar passar pela massa de grãos em um menor espaço de tempo, menor será a perda de matéria seca, consequentemente menor percentual de grãos amarelos.

Tabela 5 - Analise grãos amarelos

\begin{tabular}{|c|c|c|c|c|c|}
\hline \multicolumn{6}{|c|}{ Método } \\
\hline \multicolumn{2}{|c|}{ Hipótese nula } & \multicolumn{4}{|c|}{ Todas as médias são iguais } \\
\hline \multicolumn{2}{|c|}{ Hipótese alternativa } & \multicolumn{4}{|c|}{ Nem todas as médias são iguais } \\
\hline \multicolumn{2}{|c|}{ Nivel de significância } & \multicolumn{4}{|c|}{$\alpha=0,05$} \\
\hline \multicolumn{6}{|c|}{ Assumiu-se igualdade de variâncias para a análise } \\
\hline \multicolumn{6}{|c|}{ Informações dos Fatores } \\
\hline Fator & \multicolumn{3}{|c|}{ Niveis } & \multicolumn{2}{|c|}{ Valores } \\
\hline Ano & \multicolumn{3}{|c|}{2} & \multicolumn{2}{|c|}{$2019 ; 2020$} \\
\hline \multicolumn{6}{|c|}{ Informações de Agrupamento Usando Método de Tukey e $95 \%$ de Confianç } \\
\hline ANO & $\mathbf{N}$ & \multicolumn{2}{|c|}{ Média } & \multicolumn{2}{|c|}{ Agrupamento } \\
\hline 2019 & 11 & \multicolumn{2}{|c|}{0,1318} & A & \\
\hline 2020 & 11 & \multicolumn{2}{|c|}{0,02636} & & $\mathrm{~B}$ \\
\hline \multicolumn{6}{|c|}{ Médias que nấo compartilham uma letra são significativamente diferentes. } \\
\hline \multicolumn{6}{|c|}{ Análise de Variância } \\
\hline Fonte & GL & SQ (Aj.) & QM (Aj.) & Valor F & Valor-P \\
\hline Ano & 1 & 0,06116 & 0,061164 & 9,75 & 0,005 \\
\hline Erro & 20 & 0,12542 & 0,006271 & & \\
\hline Total & 21 & 0,18658 & & & \\
\hline
\end{tabular}

Fonte: O Pesquisador. 
Como $\mathrm{p}<\alpha$ conclui-se que o manejo de enchimento influenciou na quantidade de grãos amarelos, pois ano de 2019 foi feito enchimento parcial de cada silo, com enchimento I00\%, já no ano de 2020 foi feito enchimento parcial enchendo com $65 \%$ do silo, o que resulta em menor perca de qualidade por grãos amarelos e redução do tempo de secagem através do maior fluxo de ar.

O percentual de grãos amarelos, é o determinante de uma boa armazenagem, quanto menor, ou zero, melhor, significa que o processo de secagem acorreu corretamente. Quando produto é manejado de forma incorreta durante o processo, acaba resultando em grandes percas de qualidade, pois um ou mais grãos saios entra em processo metabólico, ocasionando uma reação química no grão em função da condição que o mesmo está exposto, estes grãos podem ficar com tonalidade amarela em todo, ou em parte do grão, podendo chegar a amarelo claro ao amarelo escuro.

Dentro do armazém, este é o indicador de maior peso, pois ele determina a qualidade do produto, mediante ao manejo adotado para ser seco e armazenado.

Abaixo temos o gráfico 2 demostrando que mediante aos percentuais de enchimento do silo, os índices de amarelos tendem a ser maiores ou menores, se comparado anos de 2019 com 2020.

Gráfico 2 - Intervalos de Grãos Amarelo x Ano

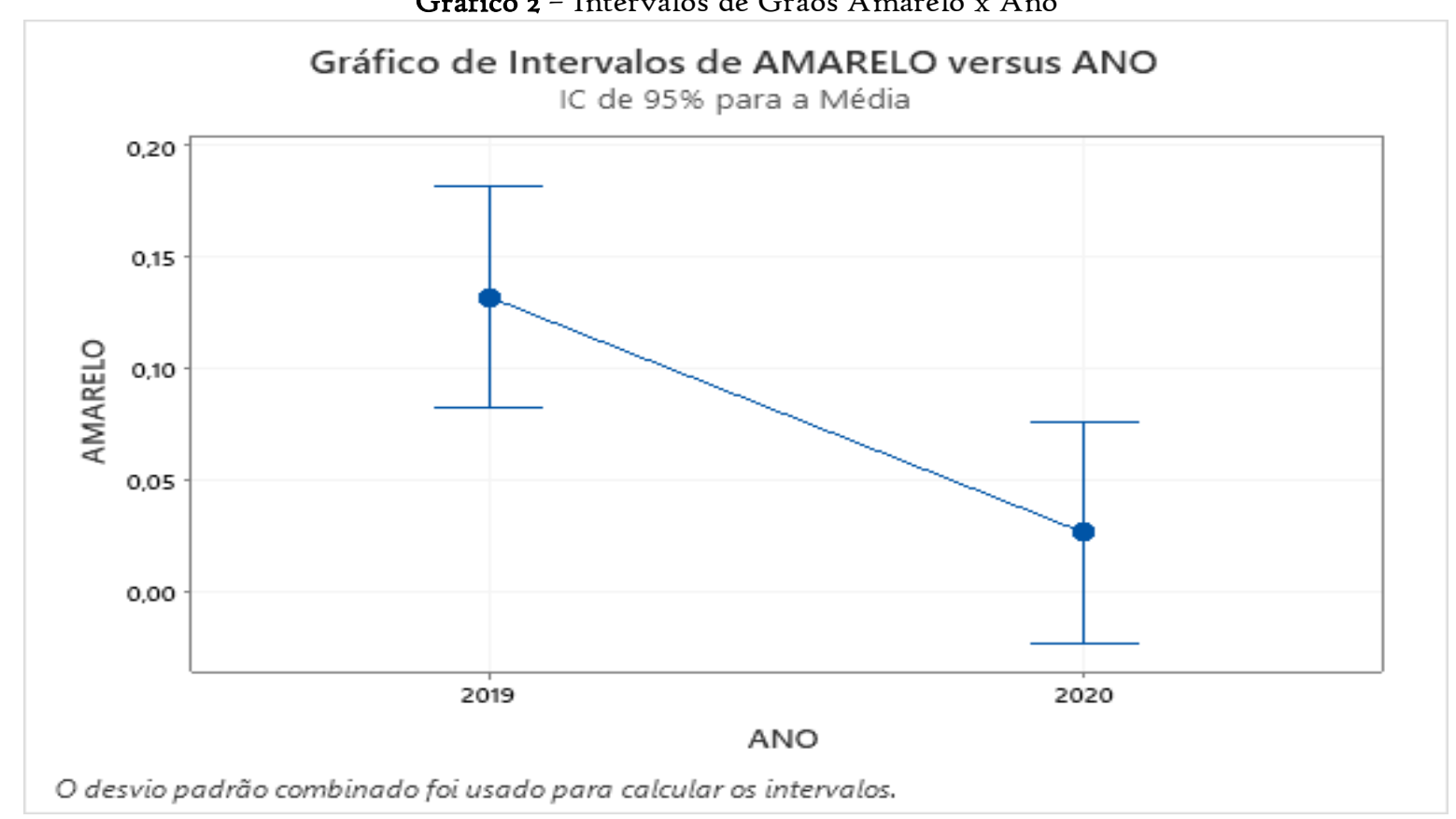

Fonte: O Pesquisador. 
4.3 Em relação ao $\mathrm{MOF} / \mathrm{ARD} / \mathrm{PR}$

ANOVA com fator de grãos $\mathrm{MOF} / \mathrm{ARD} / \mathrm{PR}$ versus o método de manejo de enchimento dos silos nos anos 2019 e 2020 não influenciou neste indicador.

Tabela 5 - Analise grãos MOF/ARD/PR

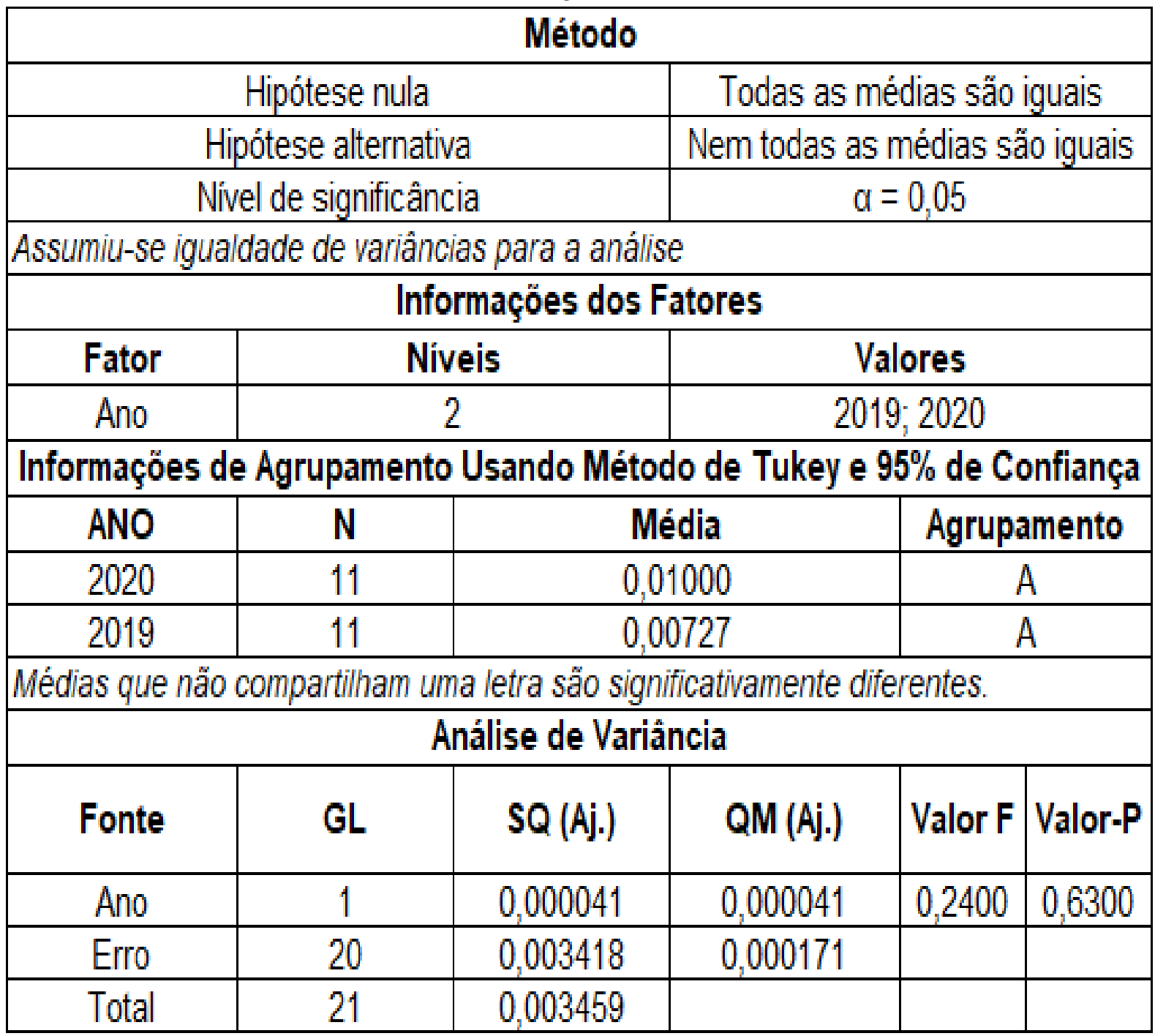

Fonte: O Pesquisador.

Como $p>\alpha$ conclui-se que o ano não influenciou no aumento significativo de grãos mofados/ardidos/pretos. No gráfico 3 podemos perceber, que a diferença neste indicador foi mínima, confirmando que o manejo adotado em cada ano não influenciou neste indicador de qualidade. 
Gráfico 3 - Intervalos de Grãos MOF/ARD/PT x Ano

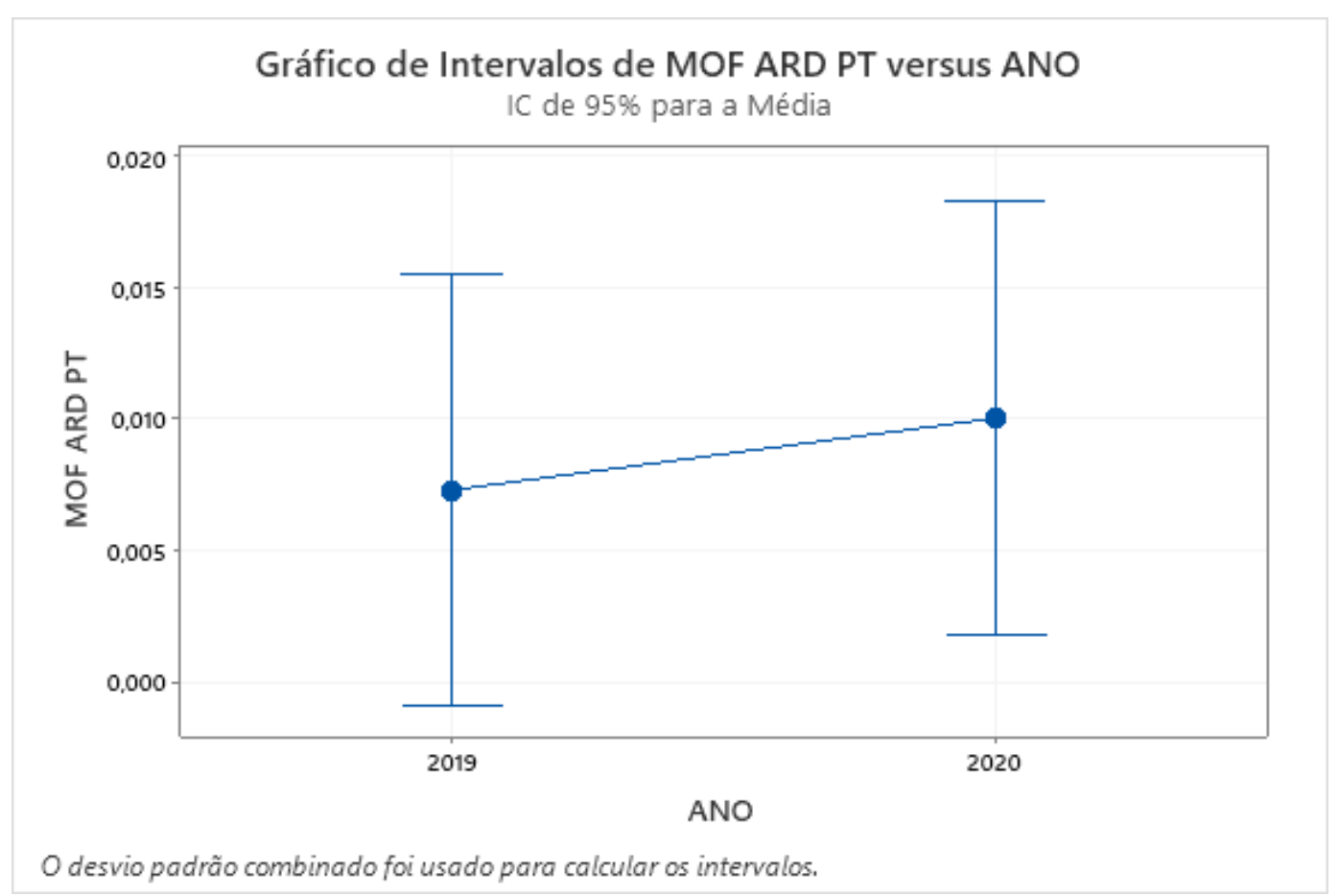

Fonte: O Pesquisador.

\section{CONCLUSÃO}

Com desenvolvimento deste trabalho para empresa em estudo aprontasse, que o manejo de enchimento do silo no momento do recebimento de cada safra de arroz implica diretamente na qualidade e velocidade de secagem do grão, através do software minitab, foi possível verificar que dependendo de como for o desenvolvimento de cada safra, por meio de enchimento parcela com $65 \%$ da capacidade do silo é possível ter uma redução no percentual de grão amarelos.

Outro ponto a ser citado, a apresentação de ferramentas estatísticas de qualidade para controlar, monitorar e validar o processo e também ao conhecimento adquirido sobre a importância de se investir o mínimo de tempo em estudos para se ter mais qualidade no processo de secagem a baixa temperatura de arroz em casca demonstrando que com manejos adequados, os resultados podem ser praticamente satisfatórios para os padrões que se deseja atingir em processamento industrial de arroz branco.

Desse modo, conclui-se que por este manejo de secagem, é possível armazenar o produto mantendo a integridade de sua qualidade. Assim, com a determinação do manejo 
adequado para enchimento, a empresa consegue ser eficaz no atendimento a seus requisitos de qualidade assegurando um produto de boa qualidade aos consumidores, contribuindo para o crescimento de toda organização.

\section{REFERÊNCIAS}

AIAG. Statistical Process Control SPC. Journal of Visual Languages \& Computing, II, 287-301.2005.

BARBOSA, S. L. O Estudo de Caso da Pesquisa em Administração: Limitações do Método ou dos Pesquisadores. In: XXXII ENANPAD, 2008, Rio de Janeiro. Anais... do XXXII ENANPAD, 2008.

BRASIL. Portaria $\mathrm{n}^{\circ}$ 6, de $\mathrm{i} 6$ de fevereiro de 2009 do Ministério da Agricultura, do Abastecimento Rural e da Reforma Agrária. Diário Oficial [da] República Federativa do Brasil, Poder Executivo, Brasília, DF, i8 fev. 2009. Seção I.

COSTA, A F. B.; EPPRECHT, E. K.; CARPINETTI, L. C. R. Controle estatístico de qualidade. São Paulo: Editora Atlas, 2004.

COSTA, A. F. B.; EPPRECHIT, E. K.; CARPINETTI, L. C. R. Controle Estatístico de 1418

Qualidade: Métodos Estatísticos. 2. ed. São Paulo: Atlas, 2005.

COSTA, A. F. B.; EPPRECHT, E. K.; CARPINETTI, L. C. R. Controle estatístico de qualidade. 2.ed, 8 reimpr. São Paulo: Atlas, 2014.

CYMROT, Raquel; MANIN, Iara Jorge; LARA, Leonardo Sgarbi. METODOLOGIA PARA IMPLANTAÇÃO DE UM LABORATÓRIO DE MINITAB, POR MEIO DA UTILIZAÇÃO DE DADOS REAIS OBTIDOS EM LEITURA DE ARTIGOS CIENTÍFICOS. In: XXXIV Congresso Brasileiro de Ensino de Engenharia. 2006.

FIFERT, C. E.; SILVA, S.R.; COSTA, V.S. Embrapa - cultivo do arroz - Normas de classificação arroz. Disponível em: <https://www.embrapa.br/cultivo-do-arroz/posproducao/normas $>$. Acesso 29 de out. 2021.

FLICK, U. Uma introdução à pesquisa qualitativa. 2. ed. Porto Alegre: Bookman, 2004. 312 p.

KOSHY, Elizabeth; KOSHY, Valsa; WATERMAN, Heather. Action research in healthcare. Sage, 2010.

LIM, S. A. H.; ANTONY, J.; ALBLIWI, S. (2014). Statistical Process Control (SPC) in the food industry - A systematic review and future research agenda. Trends in Food Science and Manual Meet Minitab, Versão I4 para Windows by Minitab Inc, 2003, 2004. 
MARTINS, Petrônio G.; LAUGENI, Fernando R.; Administração da produção. 2. ed. São Paulo: Editora Saraiva, 2005.

MACHADO, M. A. G.; COSTA, A. F. B. The use of principal components and univariate charts to control multivariate processes. Pesquisa Operacional, v. 28, n. I, p. 173196, 2008.

MELLO, C. H. P. et al. ISO 900r:2000: sistema de gestão da qualidade para operações de produção de serviços. São Paulo: Atlas, 2007.

Montgomery, D. C. (2009). Introdução ao controle estatístico da Qualidade (4a ed.). Rio de Janeiro: LTC

MONTGOMERY, D. C. Introdução ao controle estatístico da qualidade. $4^{\underline{o}}$ Ed. Rio de Janeiro: LTC, 2004.

MONTGOMERY, D. C. Introduction to Statistical Quality Control. Development, I34, 635-646. 2009 .

PABLE, A.; LU, S.; AUERBACH, J. Integrated qualitative/quantitative techniques for food product quality planning. Journal of Food Quality, 33, II2-129. Disponível em: 〈https://doi.org/ıo.IIII/j.1745-4557.2009.00287.2010〉 Acesso em 25 dez. 2021.

PIGNATIELLO JR., J.J.; SIMPSON, J.R. A magnitude-robust control chart for monitoring and estimating step changes for normal process means. Quality and Reliability Engineering International, v.18, p. 429-44I, 2002.

PIRATELLI-FILHO, A.; ZÚÑIGA, L. D. O.; ARENCIBIA, R. V. Aplicação de testes de Repetitividade e Reprodutividade (R\&R) para verificação de Scanner Laser ${ }_{3} D$. Caldas Novas: CNMAI, 2014 .

RAI, B. K. Implementation of statistical process control in an Indian tea packaging company. International Journal of Business Excellence, I(I/2), i6o.

RIBEIRO JÚNIOR, J. I. Métodos estatísticos aplicados à melhoria da qualidade. I. ed. Viçosa: UFV, 2012.

ROSA, L.C. Introdução ao Controle Estatístico de Processos. Santa Maria, Ed. da UFSM, 2009 .

Saiba mais sobre Minitab i8. Suporte ao Minitab ${ }^{\circledR}$ I8. Disponível em 〈https://support.minitab.com/pt-br/minitab/r8/getting-started/introduction/> Acesso 29 de out. 2021. 
SANTIAGO, E.; SMITH, J. Control charts based on the exponential distribution: adapting run rules for the t chart. Quality Engineering, v. 25, p. 85-96, 2013.

SHIN, H. et al. Assessment of modified Anderson-Darling test statistics for the generalized extreme value and generalized logistic distributions. Stochastic Environmental Research and Risk Assessment, [S. 1.], v. 26. n. I, p. I05-II4, jan. 2012.

SUPPORT, Minitab. Cartas de controle de variáveis no Minitab. 2021. Disponível em: 〈https://support.minitab.com/pt-br/minitab/r8/help-and-how-to/quality-and-processimprovement/control-charts/supporting-topics/understanding-variables-controlcharts/variables-control-charts-in-minitab/>. Acesso em: 28 out. 2021.

TEIXEIRA, Pietro Maxwell. Análise de nível sigma pelo software Minitab fazendo uso das etapas definir, medir e analisar empresa da ferramenta Dmaic em uma do ramo metalomecânico. 2019.

THIOLlENT, Michel. Metodologia da pesquisa-ação. ı8. ed. São Paulo: Cortez, 2011.

TOLEDO, J. C. de et al. Qualidade: Gestão e Métodos. Rio de Janeiro: LTC, 2013. 397 p.

TOLEDO, J. C. et al. Qualidade: gestão e métodos. Rio de Janeiro: LTC, 2013. 